\title{
Autophagy inhibition induces enhanced proapoptotic effects of ZD6474 in glioblastoma
}

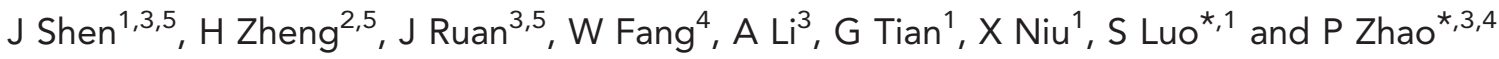 \\ ${ }^{1}$ Department of Cell Biology, Southern Medical University, Guangzhou, Gungdong, People's Republic of China; \\ ${ }^{2}$ Department of Oncology, Nanfang Hospital, Southern Medical University, Guangzhou, Guangdong, People's Republic \\ of China; ${ }^{3}$ Cancer Center, Southern Medical University, Guangzhou, Guangdong, People's Republic of China and ${ }^{4}$ Department \\ of Medical Oncology, The First Affiliated Hospital, School of Medicine, Zhejiang University, Hangzhou, Zhejiang, People's \\ Republic of China
}

Background: Autophagy is a lysosomal degradation pathway that can provide energy through its recycling mechanism to act as a cytoprotective adaptive response mediating treatment resistance in cancer cells. We investigated the autophagy-inducing effects of ZD6474, a small-molecule inhibitor that blocks activities of vascular endothelial growth factor receptor (VEGFR), epidermal growth factor receptor (EGFR), and RET tyrosine kinases.

Methods: We investigated the effects of ZD6474 on autophagy in glioblastomas cells. The ZD6474 mechanism of action was determined by western blot. We then examined the impacts of the inhibition of autophagy in combination with ZD6474 on cell apoptosis in vitro. Furthermore, we evaluated the synergistic anticancer activity of combination treatment with an autophagy inhibitor (chloroquine) and ZD6474 in U251 glioblastoma cells xenograft model.

Results: ZD6474-induced autophagy was dependent on signalling through the phosphoinositide 3-kinase/Akt/mammalian target of rapamycin (PI3K/Akt/mTOR) pathway. ZD6474-induced autophagy was inhibited by both knockdown of the ATG7 and Beclin 1 gene, essential autophagy genes, and pharmacologic agents (chloroquine and 3-methyalanine) treatment. Both treatments also dramatically sensitised glioblastoma cells to ZD6474-induced apoptosis, decreasing cell viability in vitro. Furthermore, in a xenograft mouse model, combined treatment with ZD6474 and chloroquine significantly inhibited U251 tumour growth, and increased the numbers of apoptotic cells compared with treatment with either agent alone.

Conclusion: Autophagy protects glioblastoma cells from the proapoptotic effects of ZD6474, which might contribute to tumour resistance against ZD6474 treatment.

Gliomas are the most frequently occurring brain tumours in adults. Glioblastomas belong to the most frequent and severe subtype of gliomas, and are one of the most aggressive human cancers, as demonstrated by the associated high mortality rate. The median survival of glioblastomas is only 18-21 months from the time of diagnosis when aggressive treatments are used after progression (Clark et al, 2012; Rapp et al, 2013). Insight into the molecular pathogenesis of glioblastoma has led to the recent development of targeted therapeutic strategies aimed at the interrupting key molecular signalling pathways. A large number of targeted molecular therapies are being tested alone or in combination with other therapies; such approaches include inhibitors targeting receptor tyrosine kinases such as epidermal growth factor receptor (EGFR), PDGFR, and VEGFR, as well as inhibitors of signal

\footnotetext{
*Correspondence: Dr P Zhao; E-mail: zhaop6@126.com or Professor S Luo; E-mail: luoshq888@163.com
}

${ }^{5}$ These authors contributed equally to this work

Received 13 March 2013; revised 21 May 2013; accepted 24 May 2013; published online 25 June 2013

(c) 2013 Cancer Research UK. All rights reserved 0007-0920/13 
transduction pathways directed against mTOR, PI3K, and histone. In clinical trials, these agents have shown poor or moderate effect with no increase in survival time (Gilbert, 2011). The challenge now is to develop novel therapeutic strategies to enhance these effects for glioblastoma treatment.

ZD6474 is a small-molecule inhibitor of VEGFR, EGFR, and RET tyrosine kinases that has been approved for treatment of progressive medullary thyroid cancer (Langmuir and Yver, 2012). ZD6474 inhibits the growth of numerous tumours in preclinical models (McCarty et al, 2004; Xiao et al, 2007; Beaudry et al, 2008) and has been used in recently clinical trials (Leboulleux et al, 2012; Lee et al, 2012). Moreover, recent studies have shown that ZD6474 displays activity against glioblastoma cell lines (Yiin et al, 2010), animal models (Rich et al, 2005; Navis et al, 2011) and in clinical trials (Broniscer et al, 2010; Drappatz et al, 2010). The antitumour activity of ZD6474 was potentiated in cases where ZD6474 was administered in conjunction with an mTOR inhibitor (Yang et al, 2006), the mTOR pathway negatively regulates autophagy by inhibiting formation of a trimeric complex required for autophagosome formation (Hu et al, 2012).

Autophagy is an evolutionarily conserved, intracellular selfdefense mechanism responsible for degrading unnecessary or dysfunctional cellular organelles and proteins, along with recycling cellular components to sustain cellular metabolism. Autophagy functions as a tumour suppression mechanism by isolating damaged organelles or proteins and limiting cell growth and genomic instability (Mathew et al, 2009; Yang et al, 2011). Conversely, autophagy is one mechanism that enables tumour cells to survive environmental stressors in unfavourable circumstances, as well as injuries caused by treatments such as chemotherapy, radiation therapy, and targeted therapies ( $\mathrm{Hu}$ et al, 2012b). Therefore, inhibition of autophagy may lead to increased cytotoxicity when applied in combination with anticancer drugs.

Herein, we aimed to demonstrate that ZD6474 induces autophagy by downregulating the PI3K/Akt/mTOR signalling pathway. By employing genetic and pharmacologic agents to prevent autophagy, we established that ZD6474-induced apoptosis was enhanced. These findings suggest that autophagy occurring after ZD6474 treatment likely protects glioblastoma cells from apoptosis.

\section{MATERIALS AND METHODS}

Cell cultures. Glioblastoma cell lines, U251 and U87MG, were obtained from the Cell Bank of the Chinese Academy of Sciences (Shanghai, China) and the American Type Culture Collection (Manasas, VA), respectively. The glioblastoma cells were grown in DMEM media supplemented with 5\% fetal bovine serum (FBS). ZD6474 was generously provided by AstraZeneca (Macclesfield, Cheshire, UK). Stock solutions of ZD6474 (10 mM) were dissolved in DMSO (Sigma-Aldrich, St Louis, MO, USA), stored at $-80^{\circ} \mathrm{C}$, and diluted in fresh medium immediately before use. Insulin was obtained from Sigma-Aldrich. For the treatment with an inhibitor of autophagy, cells were cultured with DMEM containing chloroquin or 3-methyalanine (3-MA) from Sigma-Aldrich plus ZD6474 or DMSO (control), and then assayed for in vitro study.

Cell viability assays. Glioblastoma cells were plated on 96-well plates (adherent cells were cultured overnight), and treated with chemicals at different concentrations. Cell viability was determined using a commercial cell counting kit (CCK-8, Dojindo, Kumamoto, Japan). In all, $10 \mu \mathrm{l}$ of CCK- 8 reagent was added to each well and incubated at $37^{\circ} \mathrm{C}$ for $3 \mathrm{~h}$ until the media appeared yellow. Absorbance was measured at $450 \mathrm{~nm}$ using a spectrophotometer. Each experiment was performed in triplicate and repeated at least three times.
Electron microscopy. Cells were fixed with $2.5 \%$ glutaraldehyde in phosphate buffer and stored at $4{ }^{\circ} \mathrm{C}$ until embedding. Cells were postfixed with $1 \%$ osmium tetroxide followed by an increasing gradient dehydration step using ethanol and acetone. Cells were then embedded in araldite, and ultrathin section was sliced $(50-60 \mathrm{~nm})$, placed on uncoated copper grids, and stained with $3 \%$ lead citrate-uranyl acetate. Images were obtained with a H600 electron microscope (Hitachi, Japan).

Transfection with fluorescent LC3 plasmid. To analyse autophagic flux, U251 cells were transfected with a pEGFP-LC3 expressing plasmid using Fugene 6, in accordance with manufacturer's instructions (Roche, Mannheim, Germany). After a 48-h transfection period, the medium was changed to DMEM $\pm \mathrm{ZD} 6474$, and the cells were further cultured and examined under an FV-1000 confocal microscope (Olympus, Tokyo, Japan).

Annexin V/PI staining. Both floating and adherent cells were collected after ZD6474 treatment $(24 \mathrm{~h})$, and were washed twice with ice-cold phosphate-buffered saline (PBS). Cells were then resuspended in $300 \mu \mathrm{l}$ of binding buffer, containing $5 \mu \mathrm{l}$ FITC-Annexin $\mathrm{V}$ and $5 \mu \mathrm{l}$ propidium iodide (PI), for $30 \mathrm{~min}$ at room temperature in the dark (Annexin V-FITC apoptosis detection kit, Nanjing Keygen Biotech, Nanjing, China). Cell survival was measured by flow cytometric analysis using a FACS Calibur flow cytometer (Becton Dickinson, San Jose, CA, USA).

RNA interference. Knockdown of ATG7 and Beclin 1 expression was achieved using transfection of small-interfering RNA (siRNA) obtained from Ambion (Norwalk, CT, USA). Oligonucleotide duplexes targeting ATG7 (s20650), Beclin 1 (s16537), and a negative control siRNA were acquired from Ambion. The siRNA was transfected into the cells using Lipofectamine 2000 (Invitrogen, Carlsbad, CA, USA) in accordance with the manufacturer's instructions.

Western blotting analysis. Cell was lysed and immunoblotted as previously described (Zhang et al, 2012). Antibodies used were as follows: phospho-S6 (S235/236), phospho-4EBP1 (T37/46), 4EBP1, phospho-Akt (S473), Akt, $\beta$-actin, HRP-conjugated anti-mouse and anti-rabbit IgG from Santa Cruz Biotechnology (Santa Cruz, CA, USA); microtubule-associated protein 1 light chain 3 (LC3), ATG7, and Beclin 1 are from Cell Signaling Technology (Beverly, MA, USA).

Caspase activity assay. Cells were plated in $175-\mathrm{cm}^{2}$ flasks. After incubation with $4 \mu \mathrm{M}$ ZD6474 for $48 \mathrm{~h}$, floating and adherent cells were harvested and combined. Preparation of cell lysates and the measurement of caspase- 9 and caspase- 3 activity were performed with Caspase- 9 and Caspase- 3 Cellular Activity Assay Kit (Calbiochem, La Jolla, CA, USA) according to manufacturer's instruction.

In vivo tumour xenograft studies. Female $\mathrm{BALB} / \mathrm{c}$ nude mice (6-8 weeks old, weighing 18-22 g) were obtained from Shanghai Slike Experimental Animals Co. (Shanghai, China; animal experimental). The animal experiments were conducted in accordance with the Guidelines for the Care and Use of Laboratory Animals. Mice were injected subcutaneously in the scapular region with $2 \times 10^{6} \mathrm{U} 251$ cells in $100 \mu \mathrm{l}$ of serum-free media. When the tumours reached a volume of $30-50 \mathrm{~mm}^{3}$, mice were randomly allocated into four groups: the groups received ZD6474 (50 $\mathrm{mg} \mathrm{kg}^{-1}$ per day; p.o.), chloroquine $\left(30 \mathrm{mg} \mathrm{kg}^{-1}\right.$ per day; i.p.), or ZD6474 + chloroquine in $100 \mu \mathrm{l}$ PBS (i.p.), and the control group received PBS (i.p.) alone. Tumours were assessed twiceweekly using a caliper to measure tumour diameter, and tumour volume was calculated according to the following formula: $V=$ $L^{2} \times W^{2} / 2(L=$ length; $W=$ width $)$. After 2 weeks of treatment, five mice per group were killed and tumours were resected for histological study. Immunohistochemical and TUNEL analysis 
were performed as previously described (Zhao et al, 2010). Ki-67 antibody (Neomarkers, Lab Vision, CA, USA) was used to measure cell proliferation, while apoptosis was analysed using an in situ cell death detection kit (TUNEL, Roche Molecular Biochemicals, Mannheim, Germany).

Statistical analysis. Results were shown as mean \pm standard error of the mean (s.e.m.). Statistical significance $(P<0.05)$ was determined by Student's $t$-test or analysis of variance (ANOVA) followed by assessment of differences using SPSS 13.0 software (SPSS Inc., Chicago, IL, USA).

\section{RESULTS}

ZD6474 activates autophagy in glioblastoma cells. To determine the effect of ZD6474 on autophagy, we used western blot analysis to observe the conversion of ATG8-related human protein LC3 from the cytoplasmic form, LC3-I, to the autophagosomic form, LC3-II, in U251 and U87MG cells after exposure to ZD6474. The LC3-II increased in a time-dependent and dose-dependent manner, indicating that autophagy might be induced by ZD6474 (Figure 1A and B). To quantify the autophagy cells, we determined the average percentage of cells with punctate fluorescence after ZD6474 treatment or not in the cells transfected with pEGFP-LC3. ZD6474-induced autophagy was demonstrated by redistribution of the autophagosome marker, GFP-LC3, from a diffuse cytoplasmic pattern to a punctate expression indicative of autophagosome formation. The U251 and U87MG cells treated with ZD6474 displayed more punctuate fluorescence than did non-treated cells (Figure 1C). Finally, ultrastructural analysis by electron microscopy confirmed that numerous autophagosomes that contained degraded materials were present in ZD6474-treated U251 and U87MG cells, but scarcely in untreated cells (Figure 1D). These data provide strong evidence that ZD6474 induces autophagy in glioblastoma cells.

ZD6474 inhibits PI3K/AKT/mTOR pathway in glioblastoma cells. The mTOR pathway is the key regulator of autophagy. To investigate the potential effect of ZD6474 on mTOR signalling in glioblastoma cells, U251 and U87MG cell lines were exposed to $4 \mu \mathrm{M}$ of ZD6474 for 2, 6, and $12 \mathrm{~h}$, components of the mTORC signalling pathway were examined by immunoblot analysis. We found that ZD6474 effectively suppressed basal levels of phosphorylation of S6 (S235/236), 4E-BP1 (T37/46), and Akt (S473) in a time-dependent manner in U251 and U87MG cells (Figure 2A). However, the expression of ATG7 and Beclin 1 was unaffected by ZD6474. To provide further evidence that the mTOR pathway is causally linked to ZD6474-induced autophagy, we analysed the autophagic response to ZD6474 in the presence or absence of insulin, a compound that strongly induces the Akt/mTOR cascade (Evert et al, 2012). The appearance of the autophagosomic form of LC3-II was correlated with the inhibition of Akt and S6 phosphorylation. Insulin increased phosphorylation of Akt and S6 in U251 cells. However, the decrease in Akt and S6 phosphorylation observed in ZD6474-treated cells was reversed by insulin; and thus, the increase in LC3-II was reversed (Figure $2 \mathrm{~B}$ ). This suggests that inhibition of the PI3K/Akt $/ \mathrm{mTOR}$ pathway is required for the induction of autophagy after ZD6474 treatment.
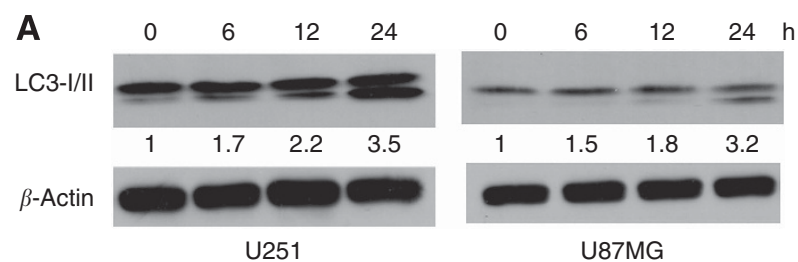

C
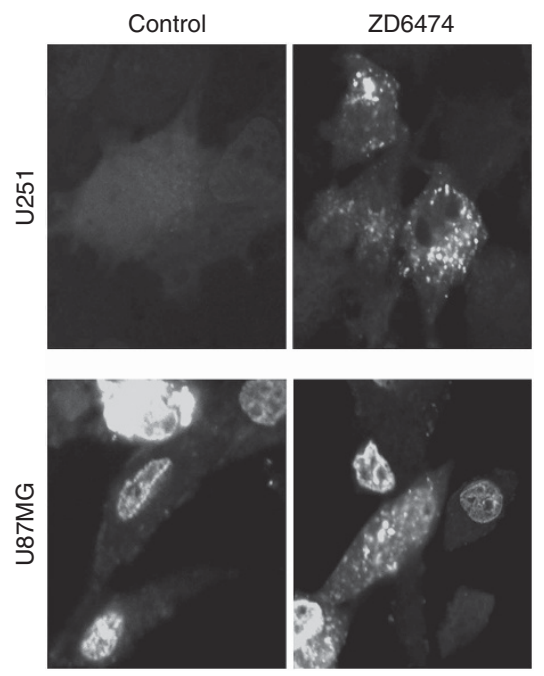
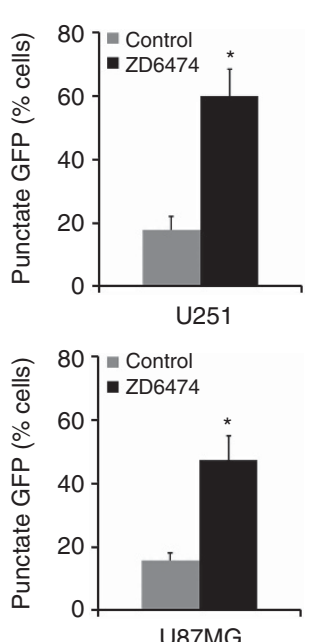

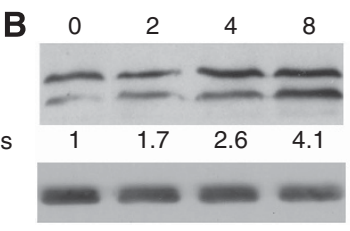

U251
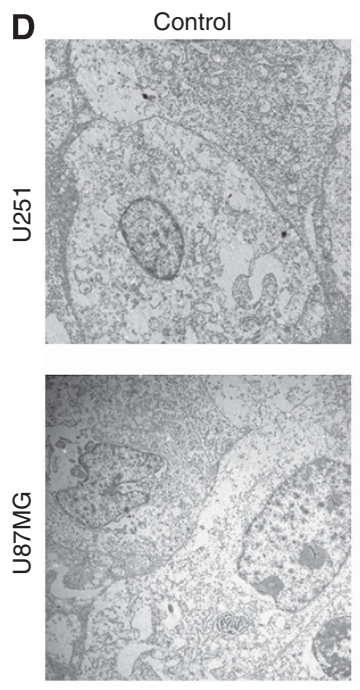
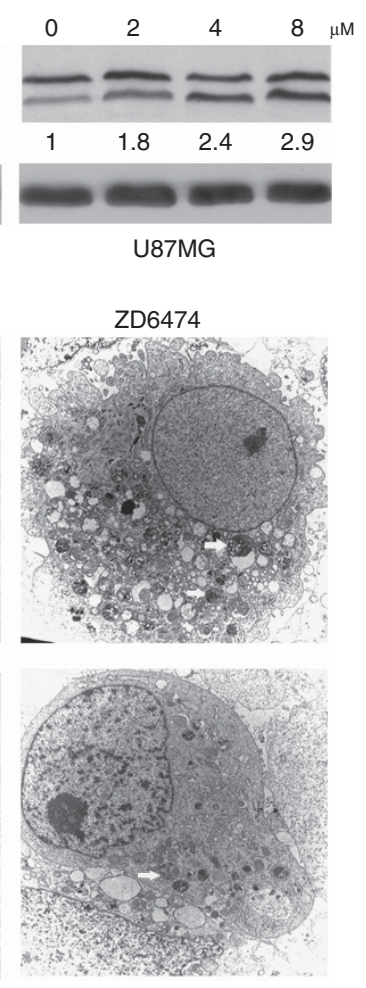

Figure 1. ZD6474 induces autophagy in glioblastoma cells. (A) Western blot showing an increase in LC3-II levels in U251 and U87MG after treatment with ZD6474. The cells were treated with or without $4 \mu \mathrm{M}$ ZD6474 for indicated time. (B) Western blot showing an increase in LC3-II levels in cells treated with 0, 2, 4, and $8 \mu \mathrm{M}$ for $12 \mathrm{~h}$. (C) Photographs of confocal microscopy of transfected GFP-LC3 plasmid in U251 and U87MG cells. Cells with punctuate GFP were counted and expressed as a percentage of the total number of cells per field. ZD6474 (4 $\mu \mathrm{m} \times 24 \mathrm{~h})$ increased the percentage of cells with punctuate green fluorescent ( ${ }^{*}<0.01$ vs non-treated cells). (D) Photographs from electron microscopy showing characteristic autophagosomes (arrows) in U251 and U87MG cells after $48 \mathrm{~h}$ of treatment with $4 \mu \mathrm{M}$ ZD6474 or no treatment. Magnification, $\times 10000$. 


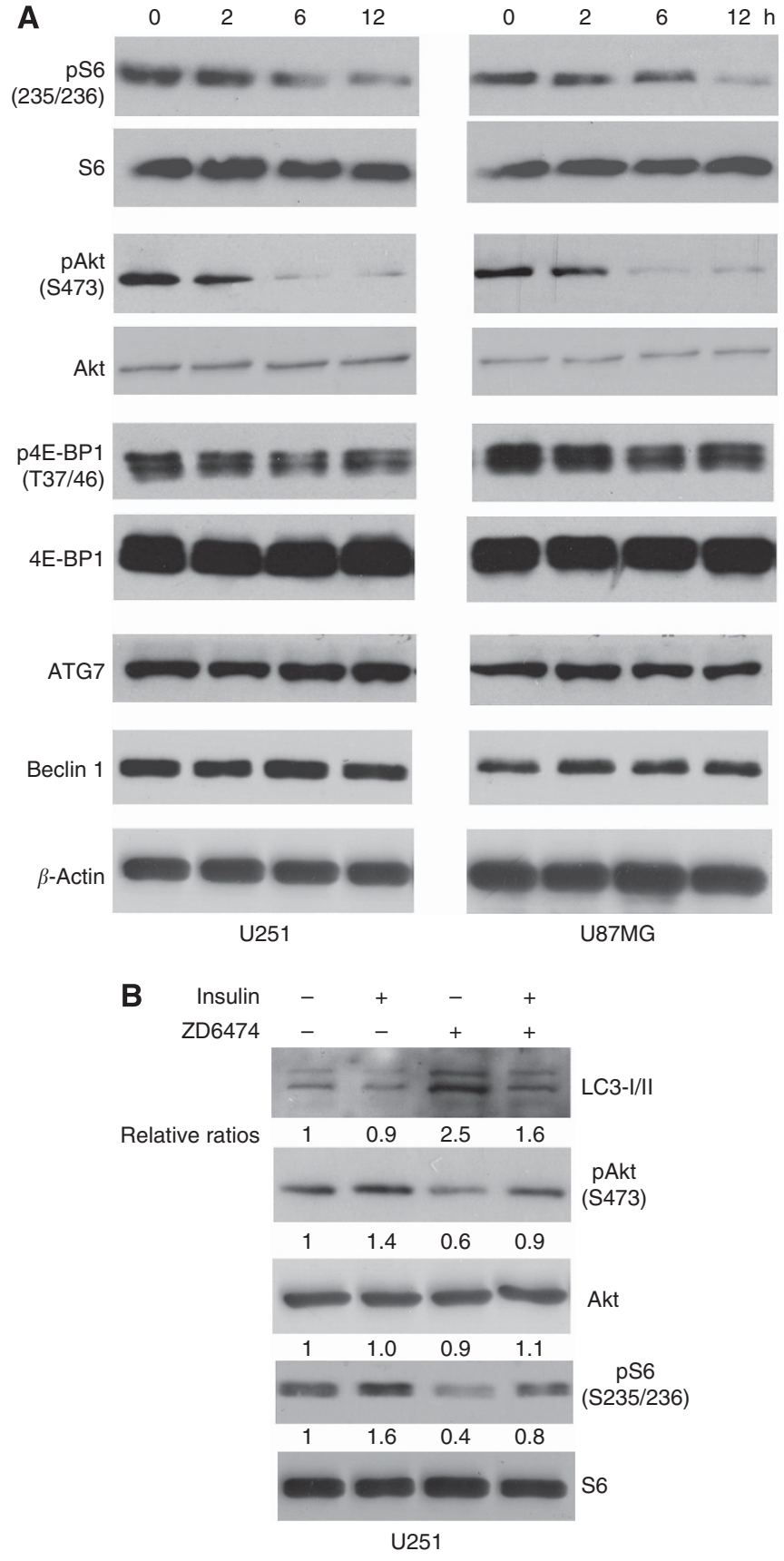

Figure 2. ZD6474 inhibits PI3K/Akt/mTOR pathway in glioblastoma cells. (A) Western blot showing decreases in S6, 4E-BP1, and AKt phosphorylation and no change in Atg7 and Beclin 1 expression. Cells were treated with $4 \mu \mathrm{m}$ ZD6474-treated cells for indicated time. The level of $\beta$-actin was used as protein-loading control. (B) Western blot showing insulin $(10 \mathrm{~nm} \times 12 \mathrm{~h})$ stimulated $\mathrm{S} 6$ phosphorylation and prevented ZD6474-induced ( $4 \mu \mathrm{M} \times 12 \mathrm{~h})$ increase in LC3-II level in U251 cells.

Inhibition of ZD6474-induced autophagy enhances the apoptotic effect of ZD6474 in vitro. To determine the biological significance of autophagy on cell apoptosis after ZD6474 treatment, we used siRNA to knockdown the expression of Atg7 and Beclin 1 in glioblastoma cells (Figure 3A). Compared with the results in siRNA controls, Atg7 knockdown prevented the ZD6474-induced increase in LC3-II levels, indicating that autophagy is clearly suppressed by ZD6474 treatment (Figure 3B). Using the method of transfection with pEGFP-LC3, we found that Atg7 knockdown of
U251 and U87MG cells showed a significant decrease in the amount of cells with punctuate green staining (Figure 3C). In addition, ZD6474 treatment in siRNA-induced Atg7 knockdown cells led to a substantial decrease in the total number of surviving cells, when compared with siRNA controls (Figure 3D). To quantify apoptosis and show the role of autophagy in protecting cells from ZD6474-induced apoptosis, we performed an Annexin V/PI staining assay to detect apoptotic cells, specifically Annexin $\mathrm{V}^{+} / \mathrm{PI}^{-}$(early apoptosis) and Annexin $\mathrm{V}^{+} / \mathrm{PI}^{+}$(late apoptosis) cells. ZD6474 treatment induced apoptosis in U251 and U87MG cells. Interestingly, Atg7 knockdown significantly enhanced the ZD6474-induced apoptosis (Figure 3E). Similar to effect of Atg7 siRNA on ZD6474-induced autophagy and apoptosis, Beclin 1 siRNA also strongly prevented autophagy (Figure $3 \mathrm{~F}$ ) and increase apoptotic cells (Figure 3G), compared with siRNA controls. This implies that autophagy has a protective role for glioblastoma cells receiving ZD6474 treatment.

To further determine the protective role of autophagy, we treated glioblastoma cells with ZD6474 in combination with the pharmacological autophagy inhibitors, chloroquine and 3MA that prevent autophagosomal degradation. Compared with the treatment with ZD6474, chloroquine or 3MA alone, treatment of glioblastoma cells with ZD6474 and chloroquine or 3MA resulted in a significant increase in the induction of apoptosis (Figure 4A). Caspase-3 and caspase-9 activity after ZD6474 treatment was clearly higher in chloroquine- or 3MA-treated cells than in the control cells (Figure 4B), indicating that inhibition of ZD6474-induced autophagy enhances the potential for apoptosis after ZD6474 treatment.

Combination treatment with ZD6474 and chloroquine caused increased apoptosis in xenografted tumour. Our results demonstrate that treatment with ZD6474, in combination with chloroquine, increased apoptosis in U251 and U87MG cells in vitro. We then investigated whether chloroquine would enhance apoptosis in a mouse xenograft tumour model. While administration of chloroquine alone did not affect tumour growth compared with PBS treatment $(P>0.05)$, ZD6474 inhibited tumour growth $(P<0.05)$. Interestingly, treatment with ZD6474, in combination with chloroquine, showed stronger inhibition of tumour growth starting from day 14 after the beginning of therapy, until the end of experiments, when compared with either agent alone (Figure 5A, $P<0.05$ ). Cell apoptosis and proliferation were evaluated using TUNEL and Ki-67 staining methods, respectively (Figure 5B). Consistent with the in vitro studies, the results of the TUNEL assay demonstrated that tumours treated with a combination of ZD6474 and chloroquine, exhibited a significant increase in apoptosis induction when compared with tumours treated with ZD6474 alone (Figure 5C, $P=0.008$ ). Conversely, treatment with ZD6474 and chloroquine did not significantly decrease Ki-67 expression when compared with treatment with ZD6474 alone (Figure 5D, $P=0.188$ ).

\section{DISCUSSION}

There is much evidence to demonstrate that tumour cells activate autophagy in response to a multitude of stressors, such as hypoxia (Hu et al, 2012a), growth factor deprivation, and damaging stimuli (White, 2012). Autophagy is an important mechanism in tumour cells for the removal of damaged organelles and aggregated proteins, and for the maintenance of energy production that leads to tumour growth and therapeutic resistance (Yang et al, 2011). Anticancer therapies including chemotherapy (Tiwari et al, 2008; Lin et al, 2012) and radiotherapy (Zhuang et al, 2011) have been found to activate autophagy in glioblastoma. Recent evidence suggests that tumour cell autophagy may act as an adaptive response mediating resistance to targeted therapy, such as 

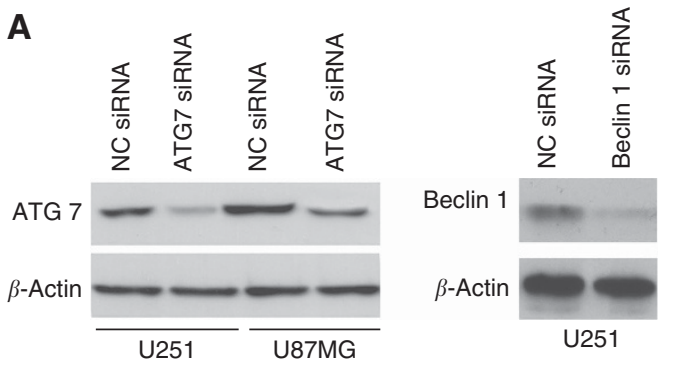

B

C

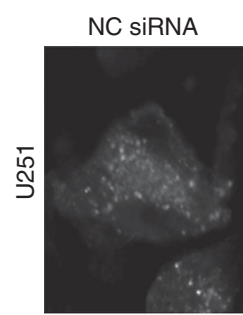

ATG7 siRNA
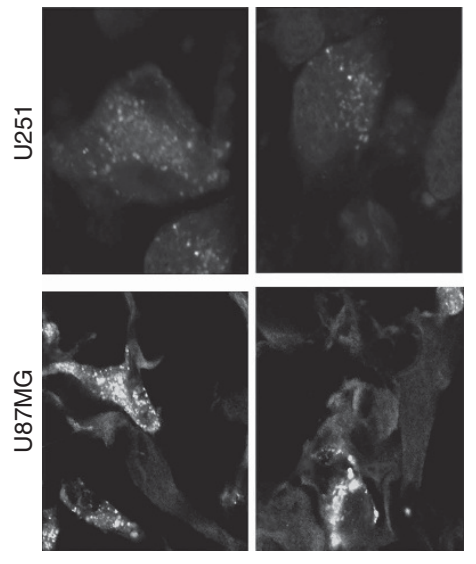

E

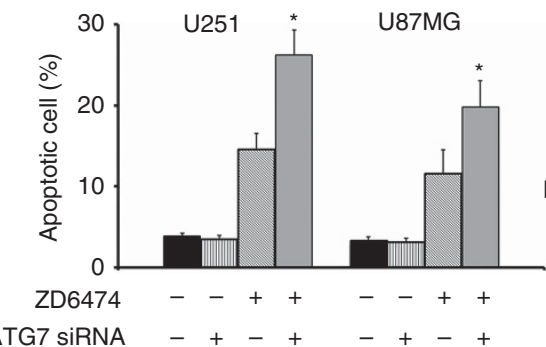

$\mathbf{F}$
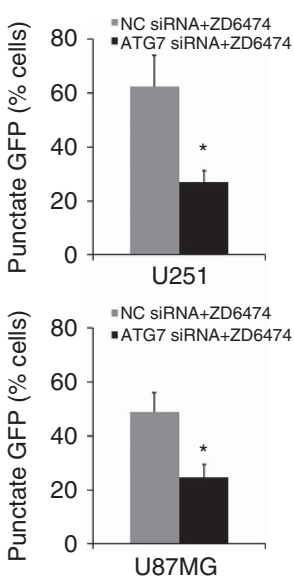

D

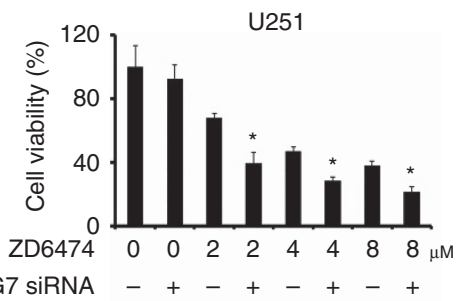

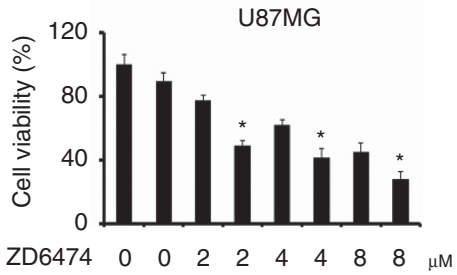

ATG7 SIRNA -+-+-+-+

G

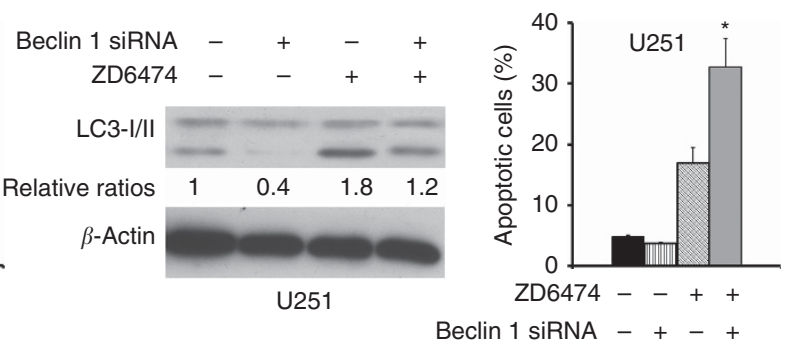

Figure 3. Effect of inhibition of Atg7 expression on ZD6474-induced apoptosis in glioblastoma cells. (A) Western blot showing decrease in the expression of Atg7 and Beclin 1, when U251 and U87MG cells were transfected with Atg7 and Beclin 1 targeting siRNA vs negative control siRNA. (B) Western blot showing that knockdown of Atg7 with siRNA prevented ZD6474-induced increase in LC3-II level in U251 and U87MG cells. (C) Photographs from confocal microscope showing the effect knockdown of Atg7 on inhibiting ZD6474-induced (4 $\mu \mathrm{M} \times 24 \mathrm{~h})$ punctuate fluorescence in U251 and U87MG cells transfected with pEGFP-LC3 expressing plasmid. Cells with punctuate GFP were counted and expressed as a percentage of the total number of cells per field. ( ${ }^{\star} P<0.01$. vs control siRNA and ZD6474). (D) Cell viability assay showing that enhanced effects of combination treatment with Atg7 siRNA treatment and indicated concentrations of ZD6474 on the survival of U251 and U87MG cells, compared with treatment with ZD6474. After $48 \mathrm{~h}$ of transfection with siRNA, the cells were treated with indicated concentrations of ZD6474 for $72 \mathrm{~h}$ $\left({ }^{\star} P<0.05\right.$ vs control siRNA and ZD6474). (E) Annexin V/PI staining assay showing that ZD6474 $(4 \mu \mathrm{M} \times 24 \mathrm{~h})$ induces higher rate of apoptosis in cells transfected with Atg7 siRNA than in that transfected with control siRNA ( ${ }^{P} P<0.05$ vs control siRNA and ZD6474). (F) Western blot showing that knockdown of Beclin 1 with siRNA prevented ZD6474-induced increase in LC3-II level in U251 cells. (G) Annexin V/PI staining assay showing that ZD6474 $(4 \mu \mathrm{M} \times 24 \mathrm{~h})$ induces higher rate of apoptosis in cells transfected with Beclin 1 siRNA than in that transfected with control siRNA $\left({ }^{\star} P<0.05\right.$ vs control siRNA and ZD6474).

sorafenib in hepatocellular carcinoma (Shimizu et al, 2012), gefitinib and erlotinib in lung cancer (Han et al, 2011), cetuximab in colorectal adenocarcinoma ( $\mathrm{Li}$ and Fan, 2010), and imatinib in chronic myeloid leukaemia (Drullion et al, 2012). To date, no studies have reported whether ZD6474 can induce autophagy. Here, we show the appearance of characteristic autophagosomes using electron microscopy, which is the gold standard for demonstrating autophagy. In addition, we show that LC3-I to LC3-II conversion and GFP-LC3 fluorescence in ZD6474-treated cells were increased in comparison with untreated cells. Together, these findings provide strong evidence to suggest that ZD6474 treatment induces autophagy in glioblastoma cells.

The mTOR pathway, a major negative regulator of autophagy, causes phosphorylation of Atg13, which reduces its interaction with ULK1 and inhibits formation of a trimeric complex required for autophagosome formation (Yang et al, 2011). The key target of mTOR complex are S6 kinase 1 (p70 S6K1) and eukaryotic initiation factor $4 \mathrm{E}$ binding protein-1 (4E-BP1). Therefore, 

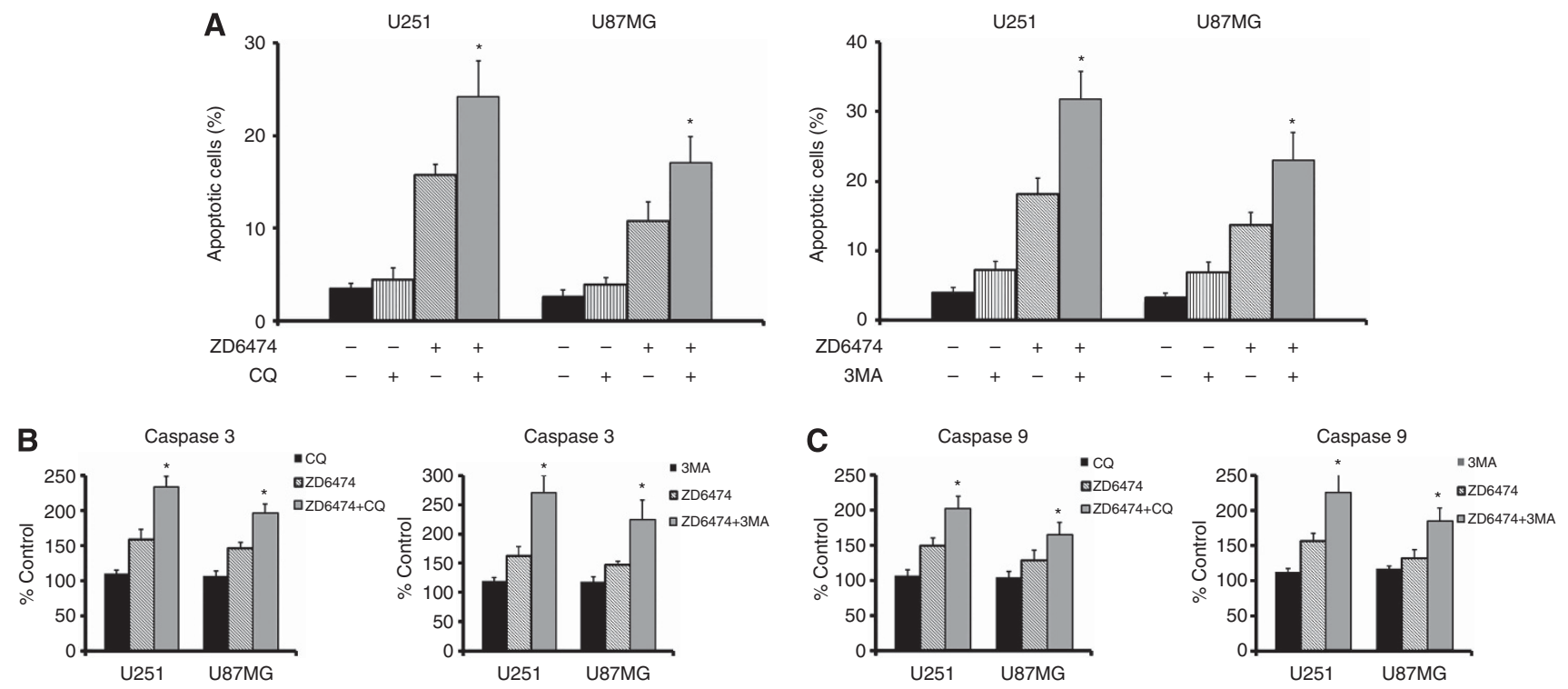

Figure 4. Chloroquine and 3-MA promote the apoptotic effect of ZD6474 in glioblastoma cells. (A) Annexin V/PI staining assay showing that combination treatment with ZD6474 $(4 \mu \mathrm{M} \times 24 \mathrm{~h})$ and chloroquine $(25 \mu \mathrm{M} \times 24 \mathrm{~h})$ or $3 \mathrm{MA}(15 \mathrm{mM} \times 24 \mathrm{~h})$ induces higher rate of apoptosis than treatment with ZD6474 only ( ${ }^{*} P<0.05$, ZD6474 plus chloroquine vs ZD6474). (B) Effect of ZD6474 treatment on the activities of caspase-3 and caspase-9. Combination therapy with ZD6474 $(4 \mu \mathrm{M} \times 48 \mathrm{~h})$ and chloroquine $(25 \mu \mathrm{M} \times 48 \mathrm{~h})$ or $3 \mathrm{MA}(15 \mathrm{mM} \times 48 \mathrm{~h})$ led to higher caspase-3 and caspase-9 activities than ZD6474 only ( ${ }^{*} P<0.05$, ZD6474 plus chloroquine vs ZD6474).

consistent with the PI3K/Akt/mTOR inhibition, the phosphorylation of its substrates including Akt, pS6 kinase (pS6K), and 4E-BP1 was decreased. We found that ZD6474 inhibited mTOR pathway as well its upstream regulator, PI3K/Akt, in glioblastoma cells. In particular, activation of PI3K/Akt/mTOR pathway by insulin inhibited ZD6474-induced autophagy. Taken together, our study suggested that PI3K/Akt/mTOR pathway might contribute to ZD6474-induced autophagy.

Autophagy is known to a double-edged sword, having a protective or a harmful role in cancer cells (Shintani and Klionsky, 2004; Wang et al, 2013). Autophagy may be an alternative mode of cell death in cancer cells that are resistant to apoptosis. When undergoing cell death and coping with excessive stress, autophagic cells may commit suicide, differing from apoptosis. Inhibition of autophagy from cytotoxic cancer therapeutics may improve antitumour activity, most likely by causing damage to DNA, cellular organelles, and proteins (Amaravadi et al, 2011). Stress-induced autophagy in tumour cells is an adaptive response mediating resistance to treatments. An abundance of preclinical evidence indicates that autophagy inhibition is currently being as a novel therapeutic strategy for anticancer therapy. The inhibition of autophagy augments the efficacy of anticancer agents in a variety of tumour in multiple preclinical models. Based on the preclinical evidence, the increasing number of investigators carried out clinical trials combining the autophagy inhibitor (chloroquine or hydroxy chloroquine) and anticancer therapies. For example, a phase III trial in glioblastoma patients treated with radiation and carmustine with or without daily chloroquine has shown chloroquine might improve mid-term survival but not quite significant, when given in addition to conventional therapy (Sotelo et al, 2006). Our findings are significant because we show that targeting autophagy through genetic early step or pharmacologic means enhances the antitumour activity of the multikinase inhibitor ZD6474 in vitro. Furthermore, using our glioblastoma cell xenograft model, we show that chloroquine dramatically potentiates the ZD6474-induced antitumour response in vivo.

Autophagy is classified as type II programmed cell death, while apoptosis is classified as type I programmed cell death. One characteristic that distinguishes apoptosis from autophagic cell death is the source of the lysosomal enzymes used for most of the dying cells' degradation. Apoptotic cells use phagocytic cell lysosomes for this process, whereas autophagic cell uses the dying cells' endogenous lysosomal machinery (Shintani and Klionsky, 2004). The cross-talk between apoptosis and autophage is complicated by the sharing regulatory and mechanistic components, such as p62/SQSTM1 (Jin et al, 2009) and Bcl-2 family proteins (Levine et al, 2008). When autophagic capacity is overwhelmed, apoptosis is promoted in cancer cells (Maiuri et al, 2007). We found that the role of autophagy in protecting glioblastoma cells from ZD6474-induced apoptosis contributes to the resistance to treatments. While caspase- 8 is essential for death receptor apoptosis, caspase- 9 is essential for the mitochondrial signalling pathways. Both pathways join at the site of caspase- 3 activation and lead to the activation of other caspases and end substrates of the apoptotic process (Jain et al, 2013). Our results show that inhibition of autophagy increases the activation of caspase- 9 and caspase-3, but does not affect the activation of caspase- 8 (data not shown). Thus, the data presented here suggest that the heightening of apoptosis by autophagy inhibitors is mediated via a mitochondrial pathway.

In summary, our findings that inhibition of autophagy at both genetic (by ATG7 and Beclin 1 knockdown) and pharmacological (chloroquine and 3-MA treatment) mean potentiated the proapoptotic effects of ZD6474 in glioblastoma cells in vitro and in vivo. The response is dependent on signalling through the $\mathrm{PI} 3 \mathrm{~K} / \mathrm{Akt} / \mathrm{mTOR}$ pathway. Our results suggest that autophagy protects cancer cells from the proapoptotic effects of ZD6474, which may be a novel target for cancer treatment with ZD6474 therapy.

\section{ACKNOWLEDGEMENTS}

We thank the staff of Department of Cell Biology, Southern Medical University for their technical assistance. This study was supported by the funding programme of National Natural Science Foundation of 


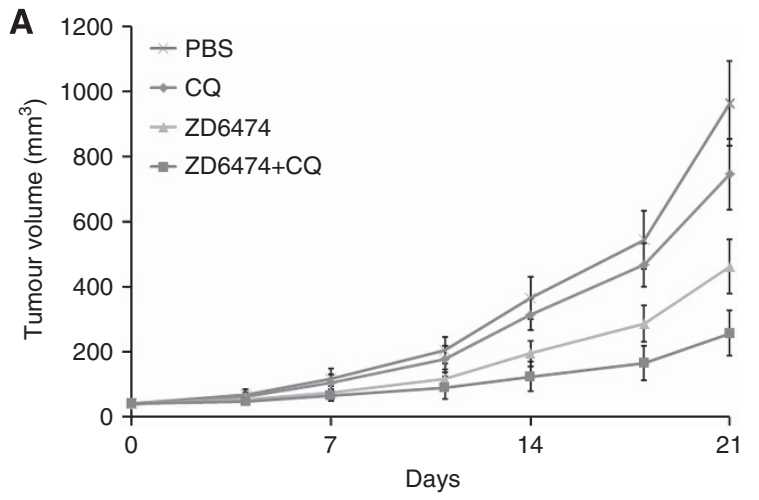

B
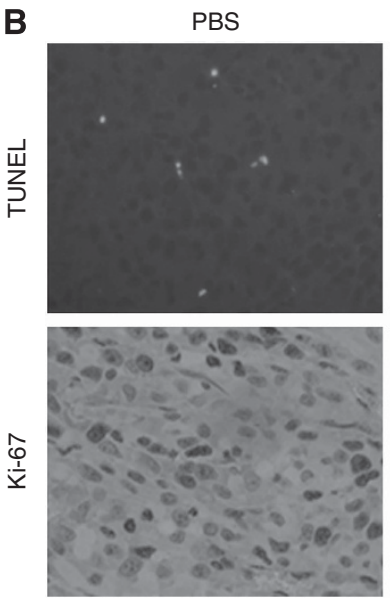

$\mathrm{CQ}$
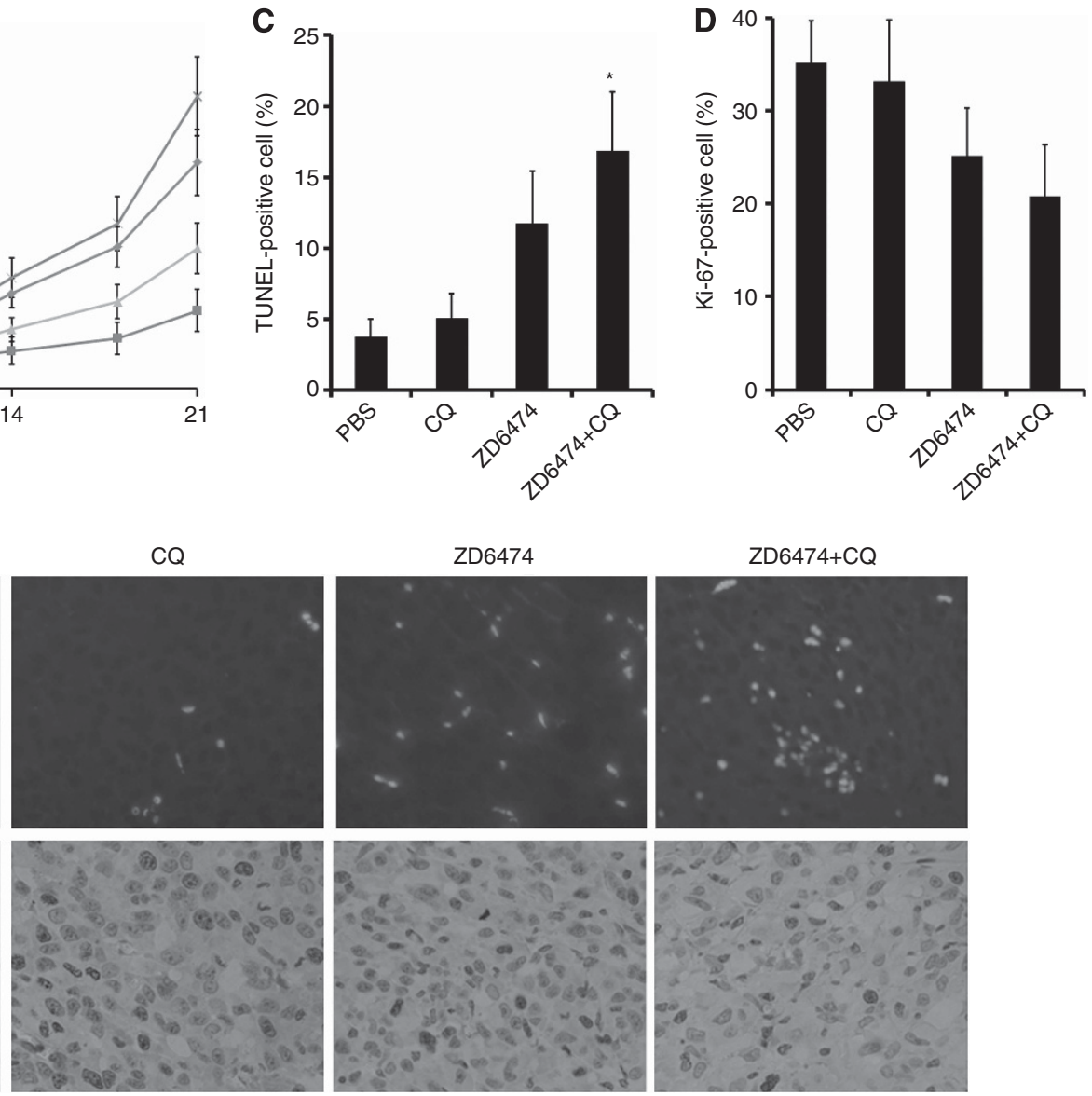

Figure 5. Inhibition of autophagy by chloroquine enhances ZD6474-induced antitumour effects in U251 xenograft tumours. (A) Xenografted tumours in nude mice were treated with PBS, chloroquine, ZD6474, and ZD6474 plus chloroquine $(n=5)$. After 2 weeks, combined therapy with ZD6474 and chloroquine inhibited tumour growth in a statistically significant manner relative to either agent alone $(P=0.04, Z D 6474$ plus chloroquine vs ZD6474; $P<0.001$, ZD6474 plus chloroquine vs chloroquine). (B) Photographs from TUNEL and Ki-67 staining in tumours received different treatment (magnification, $\times 400$ ). Proliferative cells (brown) were identified by Ki-67 staining, and apoptotic cells (green) were detected by green fluorescence. (C) Apoptotic cells increased in ZD6474-treated tumours $(P<0.001)$, and chloroquine enhanced the apoptotic effect of ZD6474 in xenograft tumours ( $P=0.008$, ZD6474 plus chloroquine vs ZD6474). (D) Proliferative cells decreased in ZD6474-treated tumours $(P=0.005)$, whereas chloroquine did not enhance the antiproliferative effect of ZD6474 in xenograft tumours $(P=0.188)$. A full colour version of this figure is available at the British Journal of Cancer journal online.

China (81001212), the Science and Technology Foundation of Zhejiang Province (2012C33011 and 2012C13014-2), and Natural Science Foundation of Guangdong (10151051501000040).

\section{CONFLICT OF INTEREST}

The authors declare no conflict of interest.

\section{REFERENCES}

Amaravadi RK, Lippincott-Schwartz J, Yin XM, Weiss WA, Takebe N, Timmer W, DiPaola RS, Lotze MT, White E (2011) Principles and current strategies for targeting autophagy for cancer treatment. Clin Cancer Res 17(4): 654-666.

Beaudry P, Nilsson M, Rioth M, Prox D, Poon D, Xu L, Zweidler-Mckay P, Ryan A, Folkman J, Ryeom S, Heymach J (2008) Potent antitumor effects of ZD6474 on neuroblastoma via dual targeting of tumor cells and tumor endothelium. Mol Cancer Therap 7(2): 418-424.

Broniscer A, Baker JN, Tagen M, Onar-Thomas A, Gilbertson RJ, Davidoff AM, Pai Panandiker AS, Leung W, Chin TK, Stewart CF, Kocak M, Rowland C, Merchant TE, Kaste SC, Gajjar A (2010) Phase I study of vandetanib during and after radiotherapy in children with diffuse intrinsic pontine glioma. J Clin Oncol 28(31): 4762-4768.

Clark AJ, Lamborn KR, Butowski NA, Chang SM, Prados MD, Clarke JL, McDermott MW, Parsa AT, Berger MS, Aghi MK (2012) Neurosurgical management and prognosis of patients with glioblastoma that progresses during bevacizumab treatment. Neurosurgery 70(2): 361-370.

Drappatz J, Norden AD, Wong ET, Doherty LM, Lafrankie DC, Ciampa A, Kesari S, Sceppa C, Gerard M, Phan P, Schiff D, Batchelor TT, Ligon KL, Young G, Muzikansky A, Weiss SE, Wen PY (2010) Phase I study of vandetanib with radiotherapy and temozolomide for newly diagnosed glioblastoma. Int J Radiat Oncol Biol Phys 78(1): 85-90.

Drullion C, Tregoat C, Lagarde V, Tan S, Gioia R, Priault M, DjavaheriMergny M, Brisson A, Auberger P, Mahon FX, Pasquet JM (2012) Apoptosis and autophagy have opposite roles on imatinib-induced K562 leukemia cell senescence. Cell Death Dis 3: e373.

Evert M, Calvisi DF, Evert K, De Murtas V, Gasparetti G, Mattu S, Destefanis G, Ladu S, Zimmermann A, Delogu S, Thiel S, Thiele A, Ribback S, Dombrowski F (2012) V-AKT murine thymoma viral oncogene homolog/ mammalian target of rapamycin activation induces a module of metabolic changes contributing to growth in insulin-induced hepatocarcinogenesis. Hepatology 55(5): 1473-1484.

Gilbert MR (2011) Recurrent glioblastoma: a fresh look at current therapies and emerging novel approaches. Semin Oncol 38(Suppl 4): S21-S33.

Han W, Pan H, Chen Y, Sun J, Wang Y, Li J, Ge W, Feng L, Lin X, Wang X, Jin $H$ (2011) EGFR tyrosine kinase inhibitors activate autophagy as a 
cytoprotective response in human lung cancer cells. PLoS ONE 6(6): e18691.

Hu YL, DeLay M, Jahangiri A, Molinaro AM, Rose SD, Carbonell WS, Aghi MK (2012a) Hypoxia-induced autophagy promotes tumor cell survival and adaptation to antiangiogenic treatment in glioblastoma. Cancer Res 72(7): 1773-1783.

Hu YL, Jahangiri A, Delay M, Aghi MK (2012) Tumor cell autophagy as an adaptive response mediating resistance to treatments such as antiangiogenic therapy. Cancer Res 72(17): 4294-4299.

Jain MV, Paczulla AM, Klonisch T, Dimgba FN, Rao SB, Roberg K, Schweizer F, Lengerke C, Davoodpour P, Palicharla VR, Maddika S, Los M (2013) Interconnections between apoptotic, autophagic and necrotic pathways: implications for cancer therapy development. J Cell Mol Med 17(1): $12-29$.

Jin Z, Li Y, Pitti R, Lawrence D, Pham VC, Lill JR, Ashkenazi A (2009) Cullin3-based polyubiquitination and p62-dependent aggregation of caspase-8 mediate extrinsic apoptosis signaling. Cell 137(4): 721-735.

Langmuir PB, Yver A (2012) Vandetanib for the treatment of thyroid cancer. Clin Pharmacol Therap 91(1): 71-80.

Leboulleux S, Bastholt L, Krause T, de la Fouchardiere C, Tennvall J, Awada A, Gomez JM, Bonichon F, Leenhardt L, Soufflet C, Licour M, Schlumberger MJ (2012) Vandetanib in locally advanced or metastatic differentiated thyroid cancer: a randomised, double-blind, phase 2 trial. Lancet Oncol 13(9): 897-905.

Lee JS, Hirsh V, Park K, Qin S, Blajman CR, Perng RP, Chen YM, Emerson L, Langmuir P, Manegold C (2012) Vandetanib Versus placebo in patients with advanced non-small-cell lung cancer after prior therapy with an epidermal growth factor receptor tyrosine kinase inhibitor: a randomized, double-blind phase III trial (ZEPHYR). J Clin Oncol 30(10): 1114-1121.

Levine B, Sinha S, Kroemer G (2008) Bcl-2 family members: dual regulators of apoptosis and autophagy. Autophagy 4(5): 600-606.

Li X, Fan Z (2010) The epidermal growth factor receptor antibody cetuximab induces autophagy in cancer cells by downregulating HIF-1alpha and Bcl-2 and activating the beclin 1/hVps34 complex. Cancer Res 70(14): 5942-5952.

Lin CJ, Lee CC, Shih YL, Lin CH, Wang SH, Chen TH, Shih CM (2012) Inhibition of mitochondria- and endoplasmic reticulum stress-mediated autophagy augments temozolomide-induced apoptosis in glioma cells. PLoS ONE 7(6): e38706.

Maiuri MC, Zalckvar E, Kimchi A, Kroemer G (2007) Self-eating and selfkilling: crosstalk between autophagy and apoptosis. Nat Rev Mol Cell Biol 8(9): 741-752.

Mathew R, Karp CM, Beaudoin B, Vuong N, Chen G, Chen HY, Bray K, Reddy A, Bhanot G, Gelinas C, Dipaola RS, Karantza-Wadsworth V, White E (2009) Autophagy suppresses tumorigenesis through elimination of p62. Cell 137(6): 1062-1075.

McCarty MF, Wey J, Stoeltzing O, Liu W, Fan F, Bucana C, Mansfield PF, Ryan AJ, Ellis LM (2004) ZD6474, a vascular endothelial growth factor receptor tyrosine kinase inhibitor with additional activity against epidermal growth factor receptor tyrosine kinase, inhibits orthotopic growth and angiogenesis of gastric cancer. Mol Cancer Therap 3(9): 1041-1048.

Navis AC, Hamans BC, Claes A, Heerschap A, Jeuken JW, Wesseling P, Leenders WP (2011) Effects of targeting the VEGF and PDGF pathways in diffuse orthotopic glioma models. J Pathol 223(5): 626-634.

Rapp M, Goeppert M, Felsberg J, Steiger HJ, Sabel M (2013) The impact of sequential vs. combined radiochemotherapy with temozolomide, resection and MGMT promoter hypermethylation on survival of patients with primary glioblastoma- a single centre retrospective study. $\mathrm{Br}$ J Neurosurg; doi:10.3109/02688697.2013.767317.
Rich JN, Sathornsumetee S, Keir ST, Kieran MW, Laforme A, Kaipainen A, McLendon RE, Graner MW, Rasheed BK, Wang L, Reardon DA, Ryan AJ, Wheeler C, Dimery I, Bigner DD, Friedman HS (2005) ZD6474, a novel tyrosine kinase inhibitor of vascular endothelial growth factor receptor and epidermal growth factor receptor, inhibits tumor growth of multiple nervous system tumors. Clin Cancer Res 11(22): 8145-8157.

Shimizu S, Takehara T, Hikita H, Kodama T, Tsunematsu H, Miyagi T, Hosui A, Ishida H, Tatsumi T, Kanto T, Hiramatsu N, Fujita N, Yoshimori T, Hayashi N (2012) Inhibition of autophagy potentiates the antitumor effect of the multikinase inhibitor sorafenib in hepatocellular carcinoma. Int $J$ Cancer 131(3): 548-557.

Shintani T, Klionsky DJ (2004) Autophagy in health and disease: a doubleedged sword. Science 306(5698): 990-995.

Sotelo J, Briceno E, Lopez-Gonzalez MA (2006) Adding chloroquine to conventional treatment for glioblastoma multiforme: a randomized, double-blind, placebo-controlled trial. Ann Intern Med 144(5): 337-343.

Tiwari M, Bajpai VK, Sahasrabuddhe AA, Kumar A, Sinha RA, Behari S, Godbole MM (2008) Inhibition of N-(4-hydroxyphenyl)retinamideinduced autophagy at a lower dose enhances cell death in malignant glioma cells. Carcinogenesis 29(3): 600-609.

Wang Z, Zhang J, Wang Y, Xing R, Yi C, Zhu H, Chen X, Guo J, Guo W, Li W, Wu L, Lu Y, Liu S (2013) Matrine, a novel autophagy inhibitor, blocks trafficking and the proteolytic activation of lysosomal proteases. Carcinogenesis 34(1): 128-138.

White E (2012) Deconvoluting the context-dependent role for autophagy in cancer. Nat Rev Cancer 12(6): 401-410.

Xiao X, Wu J, Zhu X, Zhao P, Zhou J, Liu QQ, Zheng L, Zeng M, Liu R, Huang W (2007) Induction of cell cycle arrest and apoptosis in human nasopharyngeal carcinoma cells by ZD6474, an inhibitor of VEGFR tyrosine kinase with additional activity against EGFR tyrosine kinase. Int $J$ Cancer 121(9): 2095-2104.

Yang Y, Ikezoe T, Nishioka C, Taguchi T, Zhu WG, Koeffler HP, Taguchi H (2006) ZD6474 induces growth arrest and apoptosis of GIST-T1 cells, which is enhanced by concomitant use of sunitinib. Cancer Sci 97(12): 1404-1409.

Yang ZJ, Chee CE, Huang S, Sinicrope FA (2011) The role of autophagy in cancer: therapeutic implications. Mol Cancer Therap 10(9): 1533-1541.

Yiin JJ, Hu B, Schornack PA, Sengar RS, Liu KW, Feng H, Lieberman FS, Chiou SH, Sarkaria JN, Wiener EC, Ma HI, Cheng SY (2010) ZD6474, a multitargeted inhibitor for receptor tyrosine kinases, suppresses growth of gliomas expressing an epidermal growth factor receptor mutant, EGFRvIII, in the brain. Mol Cancer Therap 9(4): 929-941.

Zhang Y, Xu S, Lin J, Yao G, Han Z, Liang B, Zou Z, Chen Z, Song Q, Dai Y, Gao T, Liu A, Bai X (2012) mTORC1 is a target of nordihydroguaiaretic acid to prevent breast tumor growth in vitro and in vivo. Breast Cancer Res Treat 136(2): 379-388.

Zhao P, Luo R, Wu J, Xie F, Li H, Xiao X, Fu L, Zhu X, Liu R, Zhu Y, Liang Z, Huang W (2010) E10A, an adenovirus carrying human endostatin gene, in combination with docetaxel treatment inhibits prostate cancer growth and metastases. J Cell Mol Med 14(1-2): 381-391.

Zhuang W, Li B, Long L, Chen L, Huang Q, Liang Z (2011) Induction of autophagy promotes differentiation of glioma-initiating cells and their radiosensitivity. Int J Cancer 129(11): 2720-2731.

This work is published under the standard license to publish agreement. After 12 months the work will become freely available and the license terms will switch to a Creative Commons AttributionNonCommercial-Share Alike 3.0 Unported License. 